\title{
IRREDUNDANT SETS OF POSTULATES FOR THE LOGIC OF PROPOSITIONS*
}

\author{
BY B. A. BERNSTEIN
}

1. Introduction. Alonzo Church $\dagger$ has introduced the interesting notion of "irredundance" in connection with a set of postulates. A set of postulates is irredundant "if the postulates are independent and no one of them can be weakened with respect to the set." I give in this paper a number of irredundant sets of postulates for the logic of propositions. $\ddagger$

Church gives a "mechanical" method by which any set of independent postulates can be made irredundant: if

$$
A, B, C
$$

is a set of independent postulates, the set

$$
A \text {, if } A \text { then } B \text {, if } A \text { and } B \text { then } C
$$

is equivalent to (I) and is irredundant. My postulate sets below have the form (II). But they are free from the irrelevances that (II) usually has when obtained mechanically by Church's rule. The postulates are simple, and in every case the hypothesis is necessary to the conclusion.

There should, of course, be no objection to an irredundant set of postulates simply because it is in form (II) $\S$ For, as Church has shown, $\uparrow$ any irredundant postulate set is equivalent (postulate for postulate) to an irredundant set of form (II). In fact, it can easily be shown that if postulate

* Presented to the Society, San Francisco Section, June 2, 1928.

$\dagger$ Alonzo Church, On irredundant sets of postulates, Transactions of this Society, vol. 27 (1925), p. 318.

$\ddagger$ For the nature of the logic of propositions see B. A. Bernstein, Sets of postulates for the logic of propositions, Transactions of this Society, vol. 28 (1926), p. 472. I shall refer to this paper in succeeding footnotes as Paper I.

\& Compare H. M. Gehman, this Bulletin, vol. 32 (1926), p. 159.

I Alonzo Church, this Bulletin, vol. 32 (1926), p. 626. 
set (I) is irredundant, then set (II) is irredundant and is equivalent to (I), postulate for postulate.* This being so, not only should there be no objection to (II) as a form for irredundant postulates, but (II) should be a desired normal form for such postulates. When a set of postulates is in this form, its irredundance can be established by simply exhibiting the necessary independence systems. My postulate sets below are, then, in the normal irredundant form, and they have been so chosen as to be simple and free from the irrelevances usually found in a set blindly made irredundant by Church's mechanical method.

I give three sets of postulates: set $\mathrm{A}$, set $\mathrm{B}$, and set $\mathrm{C}$. The undefined notions in these sets are, respectively,

$$
K, \oplus, \odot ; K, \mid ; K, \oplus .
$$

These undefined notions have been used by me before in nonirredundant sets of postulates for the logic of propositions. $\dagger$ For each set I give the necessary independence systems. Since the postulates are in the normal irredundant form, the independence systems for a set of postulates are also irredundance systems for that set and, together with a consistency system, constitute its "complete existential theory."

2. Set A. Postulates in Terms of Addition and Multiplication. The set of postulates 1-3 below is the irredundized form of my former set I. $\ddagger$ The undefined notions for this set are $K, \oplus, \odot$.

1. There is a unique $K$-element 0 such that, for every $K$-element $a$,

$$
0 \oplus a=a, \quad 0 \odot a=0 .
$$

* Church showed that the postulates (I) $A, B, C$ are equivalent respectively to (III) $A$, if $A$ then $A B$, if $A$ and $A B$ then $A B C$. Set (III) has redundancies which (II) has not.

$\dagger$ Paper I. The sets referred to are numbered I, II, III. Set IV of that paper, consisting of but a single postulate, is of course (trivially) irredundant (and "completely independent").

$\ddagger$ See Paper I. 
2. If the element 0 of Postulate 1 exists, there is a unique $K$-element $1 \neq 0$ such that, for every $K$-element $a$,

$$
1 \oplus a=1, \quad 1 \odot a=a .
$$

3. If the $K$-elements 0,1 of Postulates 1 and 2 exist, they are the only $K$-elements.

3. Irredundance of Set A. Postulate set A is in the normal irredundant form. Hence the following independence systems $(K, \oplus, \odot)$ prove the irredundance of the set. In these systems, the elements 0,1 are the Boolean zero and the whole respectively; $e^{\prime}$ is the logical negative of $e ; a+b$ and $a b$ are the logical sum and logical product respectively.
1. $K: 0,1$;
$a \oplus b=1$
$a \odot b=a b$.
$\overline{2}$. $K: 0,1$;
$a \oplus b=a b$
$a \odot b=1$.
$\overline{3} . K: 0,1, e, e^{\prime} ; a \oplus b=a+b$
$a \odot b=a b$.

4. Set B. Postulates in Terms of Rejection. Postulates $1^{\prime}-3^{\prime}$ following are the postulates of my former set $\mathrm{II}^{*}$ recast in an irredundant form. The notions left undefined are $K, \mid \cdot(a \mid b$ may be read: "not- $a$ and not- $b$ ").

$1^{\prime} . K$ consists of two elements.

$2^{\prime}$. If $K$ consists of two elements and $a$ is one of the elements, then $a \mid a$ is the other element.

$3^{\prime}$. If $K$ consists of two elements, and $a$ is one of the elements and $a \mid a$ the other, then $a \mid(a \mid a)$ and $(a \mid a) \mid a$ are one and the same $K$-elements.

5. Irredundance of Set B. The following independence systems prove the irredundance of set $B$. The elements and the operations are all Boolean.

$$
\begin{array}{ll}
\overline{1^{\prime}} . & K: 0 ; \quad a \mid b=0 . \\
\overline{2^{\prime}} . & K: 0,1 ; a \mid b=a b . \\
\overline{3^{\prime}} . & K: 0,1 ; a \mid b=b^{\prime} .
\end{array}
$$

6. Set C. Postulates in Terms of Addition. Postulates $1^{\prime \prime}-3^{\prime \prime}$ below are my former set III $\dagger$ made irredundant.

* See Paper I.

$\uparrow$ See Paper I. 
The undefined notions are $K, \oplus$.

$1^{\prime \prime}$. There is a unique $K$-element 0 such that, for every $K$-element $a$,

$$
0 \oplus a=a .
$$

$1^{\prime \prime}$. If the element 0 of Postulate 1 exists, there is a unique $K$-element $1 \neq 0$ such that, for every $K$-element $a$,

$$
1 \oplus a=1 \text {. }
$$

$3^{\prime \prime}$. If the $K$-elements 0,1 of Postulates 1 and 2 exist, they are the only $K$-elements.

7. Irredundance of Set $\mathrm{C}$. The independence systems proving the irredundance of set $C$ follow. As in the case of sets $\mathrm{A}$ and $\mathrm{B}$, the elements and the operations of these systems are all Boolean.

$$
\begin{array}{lll}
\overline{1^{\prime \prime}} . & K: 0,1 ; & a \oplus b=1 . \\
\overline{2^{\prime \prime}} . & K: 0,1 ; & a \oplus b=a^{\prime} b . \\
\overline{3^{\prime \prime}} . & K: 0,1, e, e^{\prime} ; & a \oplus b=a+b .
\end{array}
$$

8. Consistency and Sufficiency of Sets A, B, C. The following arithmetic systems* prove that sets $\mathrm{A}, \mathrm{B}, \mathrm{C}$ are consistent:
A. $K: 0,1 ; a \oplus b=a b+a+b \bmod 2 ; a \odot b=a b$.
B. $K: 0,1 ; a \mid b=a b+a+b+1 \bmod 2$.
C. $K: 0,1 ; a \oplus b=a b+a+b \bmod 2$.

The sufficiency of each of the sets A, B, C for the logic of propositions is obvious from a comparison of $\mathrm{A}, \mathrm{B}, \mathrm{C}$ with my former sets I, II, III, $\uparrow$ respectively.

The University of California

* See B. A. Bernstein, Complete sets of representations of two-element algebras, this Bulletin, vol. 30 (1924), p. 24.

$\uparrow$ See Paper I. 